\title{
Prevalência e fatores associados ao declínio cognitivo em idosos com baixa condição econômica: estudo MONIDI
}

\author{
Prevalence and factors associated with the decline in the elderly \\ with cognitive low economic condition: MONIDI study
}

Roseane Aparecida Sant' Ana do Nascimento', Rafaella Taianne Silva Batista', Saulo Vasconcelos Rocha', Lélia Renata Carneiro Vasconcelos'

\section{RESUMO}

Objetivo: Avaliar a prevalência e os fatores associados ao declínio cognitivo em idosos com baixa condição econômica. Métodos: Estudo transversal com amostra constituída de 310 idosos residentes no município de Ibicuí/BA. Foi utilizado o Miniexame do Estado Mental para a avaliação do estado cognitivo global. Para a avaliação das variáveis categóricas e análise simultânea, foram utilizados o teste qui-quadrado $\chi^{2}$ e a análise de regressão logística múltipla. A análise estatística foi realizada com nível de significância $p \leq 0,05$, cálculo da razão de chances e intervalos de confiança de 95\%. Resultados: A prevalência global do declínio cognitivo foi de 18,7\%. A análise dos dados revelou que as variáveis faixa etária e escolaridade estavam estatisticamente associadas ao maior comprometimento cognitivo entre idosos $(p \leq 0,05)$. Conclusão: A prevalência de declínio

\section{Palavras-chave} Idoso, cognição, envelhecimento.

\section{Keywords}

Elderly, cognition, aging. cognitivo observada foi elevada e associou-se a maior faixa etária e menos anos de estudo. Nesse sentido, as ações direcionadas a atenção à saúde do idoso devem fortalecer o cuidado, a prevenção e o controle das perdas cognitivas, principalmente na atenção primária.

\section{ABSTRACT}

Objective: To evaluate the prevalence and factors associated with cognitive decline in the elderly with low economic status. Methods: Cross-sectional study sample of 310 elderly residents in the city of Ibicuí/BA. We used the Mini-Mental State Examination for the assessment of global cognitive status. For the evaluation of categorical variables and simultaneous analysis was performed using chi-square test $\chi^{2}$ and multiple logistic regression analysis with exploratory purposes, respectively. Statistical analysis was performed with significance level of $p=0.05$, calculating the odds ratio and 95\% confidence intervals. Results: The overall prevalence of cognitive decline was $18.7 \%$. Data analysis revealed that the variables age and education were statistically associated with greater cognitive impairment among the elderly $(p \leq 0.05)$. Conclusion: The prevalence of cognitive decline observed was high and was associated with older age and with fewer years of schooling. In this sense, the actions directed to health care for the elderly should strengthen the care prevention and control of cognitive losses, particularly in primary care.

1 Universidade Estadual do Sudoeste da Bahia (UESB).

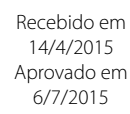

DOI: 10.1590/0047-2085000000077
Endereço para correspondência: Roseane Aparecida Sant' Ana do Nascimento

Av. José Moreira Sobrinho, 660

45206-190 - Jequié, BA, Brasil

E-mail:anne_fsaba@hotmail.com 


\section{INTRODUÇÃO}

O panorama demográfico dos países da América Latina sofreu alteração na sua estrutura etária nas últimas décadas, com acentuado aumento do contingente de idosos ${ }^{1}$. No Brasil, o aumento do número relativo de idosos contribuiu para a mudança na estrutura etária populacional ${ }^{2}$, representando 12,6\% da população geral em $2012^{3}$.

Esse cenário contribui para mudanças no perfil de morbimortalidade no país resultando no aumento da prevalência de doenças crônico-degenerativas e incapacidades, entre elas as relacionadas ao declínio cognitivo ${ }^{4}$.

No Brasil, a prevalência de declínio cognitivo (avaliado pelo Miniexame do Estado Mental) variou entre 4,9\% em Pouso Alegre/MG ${ }^{5}, 19,1 \%$ em Montes Claros/MG ${ }^{6}$ e 34,1\% em Bagé/RS?.

As perdas cognitivas são mais frequentes na população feminina ${ }^{8}$, entre indivíduos com baixa escolaridade ${ }^{9}$, que não praticam atividade física ${ }^{4}$ e também entre aqueles de baixa condição econômica ${ }^{2}$, com idade avançada ${ }^{10,11}$ e em situação de fragilidade 5 . Outros fatores de risco como doenças crônicas, entre elas hipertensão arterial'12, diabetes melito ${ }^{13}$, depressão ${ }^{14}$ e o estado de institucionalização ${ }^{9}$, aumentam a exposição às perdas cognitivas.

As alterações cognitivas muitas vezes são confundidas com processos naturais do envelhecimento, retardando seu diagnóstico e tratamento ${ }^{15}$. A detecção precoce do declínio cognitivo em idosos é uma estratégia importante. Essa informação contribui para a redução dos danos e estabelecimento de condutas terapêuticas que reduzam ou retardem a velocidade do seu aparecimento ${ }^{5}$.

As repercussões do declínio cognitivo sobre o estado geral de saúde no idoso sinalizam para a necessidade de inquéritos abrangentes sobre a situação de saúde mental, em todas as regiões do país. Dessa forma, o presente estudo tem como objetivo avaliar a prevalência e os fatores associados ao declínio cognitivo em idosos com baixa condição econômica.

\section{MÉTODOS}

Trata-se de estudo de corte transversal, realizado no município de Ibicuí/BA, no mês de fevereiro de 2014. O município de Ibicuí fica localizado na zona fisiográfica de Vitória da Conquista (Encosta do Planalto) e situado no sudoeste do estado da Bahia, com população estimada de 15.785 habitantes ${ }^{16}$. Entre eles, 1.153 com idade de 60 anos ou mais.

A população-alvo do presente estudo foi constituída por indivíduos com idade igual ou maior que 60 anos, cadastrados pela Estratégia de Saúde da Família (ESF) do município. Para a determinação do tamanho da amostra, foram utilizados os critérios propostos por Luiz e Magnanini17 para popu- lações finitas, sendo adotados: nível de significância de 5\%, intervalo de confiança de $95 \%$ e erro tolerável de 3\%. Incluíram-se $10 \%$ a mais de sujeitos na amostra para compensar possíveis perdas e recusas.

Após a aplicação dos critérios de exclusão (idosos acamados, portadores de doença de Alzheimer ou outro tipo de doença neurológica que afetasse a cognição) e contabilização das perdas (mudou-se do município, não foi encontrado por mais de três vezes na ESF ou domicílio e recusas), a amostra final foi composta por 310 idosos (201 da zona urbana e 109 da zona rural). A taxa de respostas foi de 91,2\%, com $8,8 \%(n=31)$ de recusas e 9,2\% $(n=29)$ de exclusão (Figura 1).

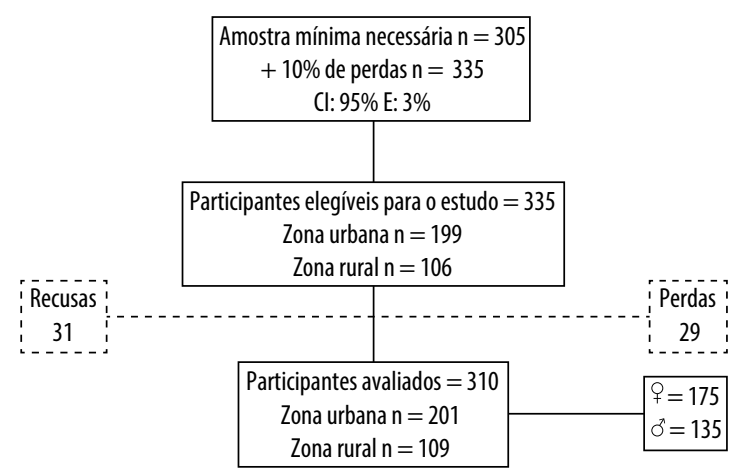

Figura 1. Organograma de distribuição da amostra do estudo.

Os dados foram coletados por meio de formulário denominado Instrumento de Avaliação da Saúde do Idoso (IASI), validado por Reis et al. ${ }^{18}$. Esse instrumento continha parte de outros questionários já validados.

Para avaliação do estado cognitivo global, foi utilizado o Miniexame do Estado Mental (MEEM) sugerido por Folstein et al. ${ }^{19}$ (1975) e validado por Bertolucci et al. em 1994 no Brasil2 ${ }^{20}$. Esse instrumento é constituído por duas seções: a primeira se refere a respostas vocais, orientação memória e atenção, com escore máximo de 21 pontos; a segunda abrange linguagem (capacidade de nomear, obedecer a comandos, escrita e desenho), com pontuação máxima de 9 pontos, totalizando o escore de 30 pontos $^{19}$. No presente estudo, foram adotados os critérios de Bertolucci et al. ${ }^{20}$ para definição do estado cognitivo global. Esses autores estabelecem pontos de corte do MEEM diferentes a depender do nível de escolaridade dos respondentes.

As variáveis independentes incluídas foram: informações pessoais e sociodemográficas - sexo (masculino e feminino), idade (em anos), escolaridade (alfabetizados e não alfabetizados), situação de moradia (acompanhado ou sozinho), renda mensal (em reais) e situação conjugal (casado, união estável, solteiro, divorciado, separado, desquitado, viúvo); hábitos de vida - consumo de bebidas alcoólicas (diariamente ou não bebe), tabaco (foi fumante, fuma atualmente, quantidade de cigarros por dia e há quanto tempo fuma), 
inatividade física no lazer e doenças referidas (hipercolesterolemia, hipertensão arterial sistêmica, doenças cardíacas e doenças circulatórias).

Para verificar a associação entre declínio cognitivo e as variáveis sociodemográficas, hábitos de vida e doenças referidas, foi utilizada medida de significância estatística para variáveis categóricas (teste qui-quadrado $\chi^{2}$ ). Para avaliação simultânea das variáveis incluídas no estudo, foi realizada análise de regressão logística múltipla, com finalidade exploratória. Foram calculados as razões de chances e respectivos intervalos de confiança (IC) de 95\%. Na análise de regressão logística, foram incluídas as variáveis que apresentaram $p \leq$ 0,20 na análise bruta. A análise dos dados foi realizada no software SPSS ${ }^{\oplus}$, versão 16.0 e R versão 2.5 .

Esta pesquisa seguiu os princípios éticos presentes na Declaração de Helsinque e na Resolução no 466/2012 do Conselho Nacional de Saúde. Os protocolos de pesquisa foram avaliados e aprovados pelo Comitê de Ética em Pesquisa com Seres Humanos da Universidade Estadual do Sudoeste da Bahia (CAAE: 22969013.0.0000.0055).

\section{RESULTADOS}

Entre os entrevistados, a média de idade foi de 71,62 \pm 8,15 anos. Observou-se maior percentual de mulheres (56,5\%), de indivíduos na faixa etária entre 60-79 anos (83,9\%), de alfabetizados $(56,1 \%)$ e de pessoas que vivem sem companheiro $(51,0 \%)$ e em estado de corresidência $(78,4 \%)$. A renda mensal média dos investigados foi de $R \$ 708,26 \pm 303,69$ reais.

No que se refere aos hábitos de vida, a maioria declarou não consumir bebidas alcoólicas regularmente $(95,8 \%)$ e relatou não fumar atualmente (88,4\%). Em relação ao nível de atividade física no lazer, $68,7 \%$ dos idosos eram inativos, ou seja, não praticavam nenhuma atividade física no tempo livre.

Quanto às doenças referidas, $13,5 \%$ dos idosos declararam-se com doença cardíaca, 64,2\%, com hipertensão arterial sistêmica, 31\%, com hipercolesterolemia e 24,8\%, com doenças circulatórias.

A prevalência global de declínio cognitivo foi de 18,7\%. A maior prevalência de declínio cognitivo foi observada entre os mais velhos (40\%), com faixa etária $\geq 80$ anos, as mulheres $(21,1 \%)$, os não alfabetizados (33,8\%), os que vivem sem companheiro $(24,7 \%)$ e os que moram sozinhos $(20,1 \%)$. Foi identificada associação com níveis estatisticamente significantes entre declínio cognitivo e faixa etária ( $p<0,001)$, escolaridade ( $p<0,001)$, situação conjugal $(p=0,006)$ e consumo de bebidas alcoólicas regularmente $(p=0,05)$ (Tabela 1 ).

No que concerne aos hábitos de vida, observa-se maior declínio cognitivo nos idosos que consumiam bebidas alcoólicas regularmente (38,5\%), nos tabagistas $(19,4 \%)$ e naqueles que não realizavam atividade física no lazer $(19,7 \%)$ (Tabela 2).
Tabela 1. Prevalência (\%) de declínio cognitivo segundo características sociodemográficas - Ibicuí/BA, 2014

\begin{tabular}{lcccc}
\hline Variáveis & $\mathrm{n}$ & $\%$ & $\chi^{2}$ & Valor dep \\
\hline Faixa etária & 38 & 14,6 & 17,76 & $<0,001$ \\
$60-79$ & 20 & 40,0 & & \\
80 ou mais & & & & \\
Sexo & 37 & 21,1 & 1,56 & 0,21 \\
Feminino & 21 & 15,6 & & \\
Masculino & 12 & 6,9 & 36,39 & $<0,001$ \\
Escolaridade & 46 & 33,8 & & \\
Alfabetizado & & & & \\
Não alfabetizado & 39 & 24,7 & 7,56 & 0,006 \\
Situação conjugal & 19 & 12,5 & & \\
Sem companheiro & & & & \\
Com companheiro & 14 & 20,9 & 0,604 \\
Situação de moradia & & & & \\
Acompanhado & 44 & 18,1 & & \\
Sozinho & 14 & & & \\
\hline
\end{tabular}

Tabela 2. Prevalência (\%) de declínio cognitivo segundo hábitos de vida - Ibicuí/BA, 2014

\begin{tabular}{lcccc}
\hline Variáveis & $\mathrm{N}$ & $\%$ & $\chi^{2}$ & Valor dep \\
\hline \multicolumn{4}{l}{ Consumo regular de bebidas alcoólicas } \\
Sim & 05 & 38,5 & 3,61 & 0,050 \\
Não & 52 & 17,6 & & \\
Tabagismo & & & & \\
Sim & 07 & 19,4 & 0,01 & 0,904 \\
Não & 51 & 18,6 & & \\
Inatividade física no lazer & & & \\
Sim & 16 & 19,7 & 0,45 & 0,500 \\
Não & 42 & 16,5 & & \\
\hline
\end{tabular}

Quanto às condições de saúde de idosos com declínio cognitivo, observou-se que 19,0\% referiram à presença de doenças cardiovasculares, 12,5\% declararam-se hipercolesterolêmicos, 19,5\% tinham doenças circulatórias e 17,1\% autodeclararam-se hipertensos (Tabela 3).

$\mathrm{Na}$ análise de regressão logística, foram incluídas as variáveis: faixa etária, escolaridade, situação conjugal, consumo regular de bebidas alcoólicas e hipercolesterolemia (variáveis que apresentaram $p \leq 0,20$ na análise bruta). Após análise multivariada, as variáveis escolaridade e faixa etária mantiveram associação com o declínio cognitivo $(p \leq 0,05)$. Indivíduos não alfabetizados e idosos longevos apresentaram maior probabilidade de apresentar perdas cognitivas quando comparados aos seus pares (Tabela 4). 
Tabela 3. Prevalência (\%) de declínio cognitivo segundo doenças referidas - Ibicuí/BA, 2014

\begin{tabular}{lcccc}
\hline Variáveis & $\mathbf{n}$ & $\%$ & $\chi^{2}$ & Valor dep \\
\hline Doenças cardíacas & & & & \\
Sim & 08 & 19,0 & 0,004 & 0,952 \\
Não & 50 & 18,7 & & \\
Hipertensão arterial sistêmica & & & \\
Sim & 34 & 17,1 & 0,96 & 0,326 \\
Não & 24 & 21,6 & & \\
Hipercolesterolemia & & & & \\
Sim & 12 & 12,5 & 3,52 & 0,060 \\
Não & 46 & 21,5 & & \\
Doenças circulatórias & & & & \\
Sim & 15 & 19,5 & 0,04 & 0,841 \\
Não & 43 & 18,5 & & \\
\hline
\end{tabular}

Tabela 4. Razão de prevalência e intervalos de confiança bruta e ajustada entre a prevalência de declínio cognitivo e variáveis do estudo - Ibicuí/ BA, 2014

\begin{tabular}{lcc}
\hline Variável & RCe IC (95\%) bruta & RCe IC (95\%) ajustada \\
\hline Faixa etária & 1,00 & 1,00 \\
$60-79$ & $1,42(1,12-1,79)$ & $1,76(1,30-1,86)$ \\
80 ou mais & & \\
Escolaridade & 1,00 & 1,00 \\
Alfabetizado & $1,40(1,23-1,59)$ & $1,82(1,65-1,91)$ \\
Não alfabetizado & & 1,00 \\
Situação conjugal & 1,00 & $1,88(0,97-3,62)$ \\
Sem companheiro & $1,97(1,19-3,25)$ & \\
Com companheiro & 1,00 & 1,00 \\
Consumo regular de bebidas alcoólicas & $1,56(0,73-3,31)$ \\
Sim & $2,1(1,05-4,54)$ & \\
Não & & 1,00 \\
Hipercolesterolemia & 1,00 & $2,87(0,75-10,93)$ \\
Sim & $1,72(0,95-3,09)$ & \\
Não &
\end{tabular}

\section{DISCUSSÃO}

A prevalência de declínio cognitivo foi elevada e associou-se à longevidade dos idosos (80 anos ou mais) e ao menor nível de escolaridade. A prevalência elevada de declínio cognitivo é um dado preocupante, em decorrência do impacto negativo das perdas cognitivas na saúde e bem-estar das pessoas idosas.

Recentes estudos em diferentes regiões brasileiras apresentam valores discrepantes quanto à prevalência de declínio cognitivo em idosos. Observaram-se prevalências

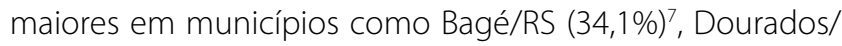

MS $(42,1 \%)^{2}$ e Viçosa/MG $(36,5 \%)^{4}$. Em outros municípios brasileiros, a ocorrência de declínio cognitivo foi mais baixa, como em Belo Horizonte/MG $(10,1 \%)^{21}$, São Paulo/SP $(6,9 \%)^{22}$ e Pouso Alegre/MG (4,9\%).

No cenário internacional, a prevalência de declínio cognitivo entre países variou mais discretamente, 27\% em idosos institucionalizados na Província de Cádiz/Espanha ${ }^{23}$, 25,9\% no estudo GERODIAB, realizado na França24 $20,1 \%$ em Xangai/China ${ }^{25}$ e 13,8\% em Guadalajara/México ${ }^{26}$.

As perdas cognitivas são morbidades que interferem negativamente na qualidade de vida dos idosos, com dificuldades de manutenção da atenção, memória recente e elaboração de cálculos ${ }^{5}$. As perdas neuronais graduais com o avançar da idade resultam na diminuição da capacidade funcional, com consequente prejuízo da memória5.

Com o avanço da idade há redução nos escores obtidos no MEEM, o que sugere pior desempenho na cognição 2,27. Lebrão e Laurenti ${ }^{22}$, durante o estudo SABE, identificaram aumento linear nas perdas cognitivas com o avançar da idade, variando de 4,2\% na faixa etária de idosos de 60-74 anos a $17,7 \%$ entre os idosos de 75 anos ou mais.

Em estudo de coorte realizado em Bambuí/MG, os autores mostraram maior incidência de declínio cognitivo, assim como declínio mais acelerado da função cognitiva, nos grupos de idosos mais velhos ${ }^{11}$.

Apesar da inevitável diminuição da função cognitiva com o aumento da idade, o prejuízo dessas perdas apenas é constatado pelo idoso ou sua família quando lhe é solicitado um esforço cognitivo maior do que o habitualmente realizado em seu cotidiano ${ }^{5}$.

Outras condições, além da longevidade, podem acentuar as perdas cognitivas. No presente estudo, os indivíduos com menores níveis de escolaridade apresentaram maior prevalência de declínio cognitivo. O aumento da escolaridade caracteriza-se como um fator de proteção em relação às perdas cognitivas.

Indivíduos com maior escolaridade teriam maiores ligações entre as distintas áreas cerebrais ${ }^{8}$. Além disso, o aumento da escolaridade estaria relacionado à maior resistência e flexibilidade ao cérebro contra patologias e alterações neuronais próprias do envelhecimento 28,29 .

A escolarização realizada desde infância até a fase adulta leva o indivíduo a se sobrepor ao conhecimento básico, partindo para a diversificação de atividades e intelectualização, melhorando o seu desempenho em testes de habilidades cognitivas durante a velhice, revelando-se como caráter protetor da cognição ${ }^{30}$.

A escolarização atua na aceleração da velocidade de processamento do raciocínio, atenção, inteligência, funções executivas e memória, retardando a perda cognitiva global e melhorando o desempenho dos idosos nos testes cogniti$\operatorname{vos}^{27}$. Idosos com menos anos de estudo apresentam probabilidade 3,83 vezes maior de ter declínio cognitivo ${ }^{31}$. 
Banhato et al..$^{32}$ mostraram que existe forte influência da variável escolaridade sobre a cognição. Os autores identificaram que, após analisar separadamente dois grupos (estratificado pela escolaridade), aqueles que apresentaram melhor pontuação no MEEM eram os idosos com maior nível de escolaridade.

É importante salientar que a observação prévia de estudos internacionais acerca da influência da escolaridade sobre os escores do MEEM levou ao ajustamento estratificado dos pontos de corte conforme os anos de educação formal apresentados pelos entrevistados nos países em desenvolvimento, em virtude do grande número de analfabetos e indivíduos com baixa escolaridade nessas populações, incluindo o Brasili33.

Neste estudo, a prevalência de declínio cognitivo foi maior entre os analfabetos, corroborando estudos nacionais e internacionais ${ }^{8}$ acerca do tema. A prevalência de comprometimento cognitivo leve em idosos de Xangai/China foi de 50,5\% entre os que não haviam completado a escola primária do país e gradativamente foi decrescendo conforme aumentaram os anos de estudos formal ${ }^{25}$. Em estudo realizado na Colômbia, identificou-se redução da prevalência de declínio cognitivo com o incremento dos anos de estudo 55,8\%: 0-4 anos e 31,8\%: 5-8 anos, respectivamente ${ }^{34}$.

Associação entre escolaridade e perdas cognitivas também foi observada em levantamento conduzido entre a população norte-americana, em que os autores constataram que idosos que viviam em áreas onde a maioria da população não tinha atingido o ensino médio apresentavam menor capacidade cognitiva. Os autores mostraram que as perdas cognitivas foram acentuadas pela exposição a condições de vida estressantes (desemprego, falta de atividades de lazer, baixa renda) ${ }^{35}$.

Entre as limitações impostas por este estudo, aponta-se seu caráter transversal, que impossibilita avaliação longitudinal das variáveis investigadas, e os resultados encontrados podem ter sido afetados pela causalidade reversa, podendo as variáveis de exposição estudadas ter sido influenciadas pelo efeito de interesse. Contudo, os resultados obtidos se apresentam como referência para a gestão em saúde, constituindo-se como elemento adicional na construção de políticas públicas de intervenção em saúde do idoso.

Ainda, deve-se levar em consideração que a avaliação do comprometimento cognitivo foi realizada por um único instrumento de triagem (o MEM), e essa situação poderia caracterizar um viés de aferição. Entretanto, o instrumento utilizado é extensamente utilizado na literatura não só no Brasil, mas de diferentes países do mundo, o que favorece a comparação dos resultados encontrados com outros estudos.

\section{CONCLUSÃO}

A prevalência de declínio cognitivo nesse estudo foi elevada. $O$ analfabetismo e a faixa etária $\geq 80$ anos aumentaram a exposição a perdas cognitivas entre os idosos. Os resultados do presente estudo indicam que, em parte, as variáveis relacionadas ao declínio cognitivo são passíveis de ações preventivas. O acesso à escolaridade, à renda e aos hábitos de vida saudáveis e o acompanhamento específico da população geriátrica na atenção primária à saúde podem influenciar positivamente na preservação da capacidade cognitiva dos idosos.

\section{CONTRIBUIÇÕES INDIVIDUAIS}

Todos os autores colaboraram expressivamente na construção e caracterização do presente estudo e na análise e interpretação dos dados. Contribuíram também na concepção do artigo, bem como revisaram criticamente o seu conteúdo intelectual e aprovaram a versão final a ser publicada.

\section{CONFLITO DE INTERESSE}

Não houve nenhum conflito de interesse.

\section{AGRADECIMENTOS}

Agradecemos ao Núcleo de Estudo em Saúde da População (NESP), que contribuiu cedendo os dados da pesquisa para a realização deste artigo, e ao Conselho Nacional de Desenvolvimento Científico e Tecnológico (CNPq) e à Fundação de Amparo à Pesquisa do Estado da Bahia (Fapesb), pela concessão de bolsa de iniciação científica à graduanda Roseane Aparecida Sant'Ana do Nascimento, no período do estudo (2014-2015).

\section{REFERÊNCIAS}

1. Organización Panamericana de la Salud. Envejecimiento y salud: un cambio de paradigma. Rev Panam Salud Publica. 2000;7(1):60-7.

2. Macêdo AML, Cerchiari EAN, Alvarenga MRM, Faccenda 0, Oliveira MAC. Avaliação funcional de idosos com déficit cognitivo. Acta Paul Enferm. 2012;25(3):358-63.

3. Instituto Brasileiro de Geografia e Estatística. Pesquisa Nacional por Amostra de Domicílios: Síntese de indicadores 2012. Disponível em: <http://biblioteca.bge.gov.br/visualizaca0/livros/liv65857.pdf>. Acesso em: 26 mar. 2015.

4. Freitas DHM, Campos FCA, Linhares LQ, Santos CR, Ferreira CB, Diniz BS, et al. Autopercepção da saúde e desempenho cognitivo em idosos residentes na comunidade. Rev Psiquiatr Clín. 2010;37(1):32-5.

5. Faria EC, Silva SA, Farias KRA, Cintra A. Avaliação cognitiva de pessoas idosas cadastradas na estratégia saúde da família: município do Sul de Minas. Rev Esc Enferm USP. 2011;45(2):1748-52.

6. Ferreira RC, Vargas AMD, Fernandes NCN, Souza JGS, Sá MAB, Oliveira LFB, et al. 0 idoso com comprometimento cognitivo apresenta pior condição de saúde bucal? Ciênc Saúde Coletiva. 2014;19(8):3417-28

7. Holz AW, Nunes BP, ThuméE, Lange C, Facchini LA. Prevalence of cognitive impairment and associated factors among the elderly in Bagé, Rio Grande do Sul, Brazil. Rev Bras Epidemiol. 2013;16(4):880-8. 
8. Santos AA, Mansano-Schlosser TCS, Ceolim MF, Pavarini SCI. Sono, fragilidade e cognição: estudo multicêntrico com idosos brasileiros. Rev Bras Enferm. 2013;66(3):351-7.

9. Trindade APNT, Barboza MA, Oliveira FB, Borges APO. Repercussão do declínio cognitivo na capacidade funcional em idosos institucionalizados e não institucionalizados. Fisioter Mov. 2013;26(2):281-9.

10. Diniz BSO, Volpe FM, Tavares AR. Nível educacional e idade no desempenho no Miniexame do Estado Mental em idosos residentes na comunidade. Rev Psiquiatr Clín. 2007;34(1):13-7.

11. Castro-Costa E, Dewey ME, Uchôa E, Firmo JOA, Lima-Costa MF, Stewart R. Trajectories of cognitive decline over 10 years in a Brazilian elderly population: the Bambuí cohort study of aging. Cad Saude Publica. 2011;27(Supl 3):345-50.

12. Banhato EFC, Guedes DV. Cognição e hipertensão: influência da escolaridade. Estud Psicol. 2011;28(2):143-51.

13. Almeida-Pititto B, Almada Filho CM, Cendoroglo MS. Déficit cognitivo: mais uma complicação do diabetes melito? Arq Bras Endocrinol Metab. 2008;52(7):1076-83.

14. Trentini CM, Werlang BSG, Xavier FMF, Argimon IIL. A relação entre variáveis de saúde mental e cognição em idosos viúvos. Psicol Reflex Crít. 2009;22(2):236-43.

15. Santos FH, Andrade VM, Bueno OFA. Envelhecimento: um processo multifatorial. Psicol Estud. 2009;14(1):3-10

16. Instituto Brasileiro de Geografia e Estatística. Síntese de indicadores sociais 2010: uma análise das condições de vida da população brasileira. Disponível em: <http://www.ibge. gov.br/home/estatistica/populacao/censo2010/indicadores_sociais_municipais/indicadores_sociais_municipais_tab_uf_zip.shtm>. Acesso em: 12 out. 2013.

17. Luiz RR, Magnanini MMF. A lógica da determinação do tamanho da amostra em investigações epidemiológicas. Cad Saúde Colet (Rio J). 2000;8(2):9-28.

18. Reis MC, Nascimento RAS, Pedreira RBS, Rocha SV, Vasconcelos LRC, Diniz KO, et al. Validação de face e clareza do Instrumento de Avaliação da Saúde dos Idosos - IASI. In: Anais do XIX Congresso Brasileiro de Geriatria e Gerontologia; 2014. p. 296.

19. Folstein MF, Folstein SE, Mchugh PR. "Mini-mental state": a practical method for grading the cognitive state of patients for the clinician. J Psychiatr Res. 1975;12(3):189-98. http:// www.ncbi.nlm.nih.gov/pubmed/1202204. Acessado em 25 de março de 2015.

20. Bertolucci PHF, Brucki SMD, Campacci SR, Juliano Y. 0 miniexame do estado mental em uma população geral: impacto da escolaridade. Arq Neuropsiquiatr. 1994;52(1):1-7.

21. Ursine PGS, Cordeiro HA, Moraes CL. Prevalência de idosos restritos ao domićlío em região metropolitana de Belo Horizonte (Minas Gerais, Brasil). Ciênc Saúde Coletiva. 2011;16(6):2953-62
22. Lebrão ML, Laurenti R. Saúde, bem-estar e envelhecimento: 0 estudo SABE no Município de São Paulo. Rev Bras Epidemiol. 2005;8(2):127-41.

23. Sánchez JMV, Palma MR. Prevalencia del deterioro cognitivo leve en mayores institucionalizados. Gerokomos. 2010;21(4):153-7.

24. Verny C, Doucet J, Bauduceau B, Constans T, Mondon K, Le Floch JP. Prevalence of cognitive decline and associated factors in elderly type 2 diabetic patients at inclusion in the GERODIAB cohort. Eur Geriatr Med. 2015;6(1):36-40.

25. Ding D, Zhao Q, Guo Q, Meng H, Wang B, Luo J, et al. Prevalence of mild cognitive impairment in an urban community in China: a cross-sectional analysis of the Shanghai Aging Study. Alzheimers Dement. 2014;1(10):1-12.

26. Ortiz GG, Arias-Merino ED, Flores-Saiffe ME, Velázquez-Brizuela IE, Macías-Islas MA, Pacheco-Moisés FP. Prevalence of cognitive impairment and depression among a population aged over 60 years in the metropolitan area of Guadalajara, Mexico. Curr Gerontol Geriatr Res. 2012;2012:1-6.

27. Domiciano BR, Braga DKAP, Silva PN, Vasconcelos TB, Macena RHM. Escolaridade, idade e perdas cognitivas de idosas residentes em instituições de longa permanência. Rev Neurocienc. 2014;22(3):330-6.

28. Coelho FGM, Vital TM, Novais IP, Costa GA, Stella F, Santos-Galduroz RF. Desempenho cognitivo em diferentes níveis de escolaridade de adultos e idosos ativos. Rev Bras Geriatr Gerontol. 2012;15(1):7-15.

29. Reis LA, Torres GV, Araújo CC, Novaes LKN. Rastreamento cognitivo de idosos institucionalizados no município de Jequié-BA. Psicol Estud. 2009;14(2):295-301.

30. Souza AAF, Wechsler SM. Inteligência e criatividade na maturidade e velhice. Psicol Reflex Crit. 2013;26(4):643-53

31. Machado JC, Ribeiro RCL, Cotta RMM, Leal PFG. Declínio cognitivo de idosos e sua associação com fatores epidemiológicos em Viçosa, Minas Gerais. Rev Bras Geriatr Gerontol. 2011;14(1):109-21.

32. Banhato EFC, Leite ICG, Guedes DV, Chaoubah A. Cognição de idosos: estudo a partir da FA8 - Forma Abreviada da Wechsler-III. Psicol Reflex Crit. 2012;25(1):96-104.

33. Borges LJ, Benedetti TRB, Mazo GZ. Rastreamento cognitivo e sintomas depressivos em idosos iniciantes em programa de exercício físico. J Bras Psiquiatr. 2007;56(4):273-9.

34. Alvarado C, Gómez JF, Etayo E, Giraldo CE, Pineda A, Toro E. Estudio EDECO Estudio poblacional de deterioro cognitivo en población colombiana. Acta Med Colomb. 2014;39(3):264-71.

35. Wight RG, Aneshensel CS, Miller-Martinez D, Botticello AL, Cummings JR, Karlamangla AS, et al. Urban neighborhood context, educational attainment, and cognitive function among older adults. Am J Epidemiol. 2006;163(12):1071-8. 